\title{
Changes in forward light scatter parameters as a function of refractive error in young adults
}

\author{
Manbir Nagra ${ }^{1}$ (D) $\cdot$ Mansi Patel $^{2} \cdot$ John Barbur ${ }^{2}$
}

Received: 17 July 2019 / Revised: 10 December 2019 / Accepted: 19 December 2019 / Published online: 7 January 2020

(C) The Author(s) 2020

\begin{abstract}
Background/aims Some aspects of visual performance worsen with increasing myopia. Whilst the underlying causes are not always clear, reduction in retinal image quality is often attributed to structural changes in the posterior myopic eye. Forward light scatter, originating principally from the cornea and lens, is known to produce veiling glare which subsequently reduces retinal image contrast. It is therefore of interest to investigate whether forward light scatter varies with refractive error.

Methods Thirteen young-adult subjects (18-25 years), with mean spherical errors (MSE $\pm \mathrm{sd}, \mathrm{D}$ ) RE, $-1.69 \pm 2.02$ (range 0.38 to -4.75 ); LE, $-1.91 \pm 1.94$ (range 0.50 to -4.63 ) underwent binocular assessment of forward light scatter using the AVOT light scatter test. Five glare annuli, with effective eccentricities ranging from 2 to $10^{\circ}$, were used to estimate parameters, $k$ and $n$, which define the light scatter function of the eye. These were then used to calculate the area under the light scatter function $\left(k^{\prime}\right)$ and the total volume of light scatter $\left(k^{\prime \prime}\right)$.

Results Significant correlation was found between increasing myopia and $k^{\prime}$ values (RE, $p<0.05 ; r=0.64$; LE, $p<0.05, r=$ 0.66). Neither the 'volume' of light scatter $\left(k^{\prime \prime}\right)$, the parameter, $n$, which controls the angular distribution of light scatter, or the straylight parameter constant, $k$, were significantly correlated with refractive error ( $p>0.05$ for both eyes). Axial length was also not correlated with any of the light scatter parameters measured.

Conclusion The preliminary data from this study provide evidence that some light scatter parameters may be correlated with refractive error. Further studies are needed to characterize how changes in the anterior media of the eye, and inclusion of a wider range of refractive errors, may affect forward light scatter.
\end{abstract}

Keywords Refractive error $\cdot$ Forward light scatter $\cdot$ Retinal straylight $\cdot$ Myopia $\cdot$ Axial length

\section{Introduction}

Some visual functions in myopes are often poorer when compared with emmetropes [1-3], even when refractive errors are fully corrected. Owing to the strong association between increasing myopia and axial length elongation, the impaired visual response is frequently attributed to structural and neural changes relating to the posterior region of the myopic eye. It has been speculated that reduced retinal function in myopia

Manbir Nagra

manbir.nagra@port.ac.uk

1 School of Health and Care Professions, University of Portsmouth, Portsmouth PO1 2DT, UK

2 Applied Vision Research Centre, Division of Optometry and Visual Science, City, University of London, London EC1V 0HB, UK may include loss of cell function [4]; reduced retinal cell density [5]; and misalignment of photoreceptors $[6,7]$.

A less well studied cause of retinal image degradation is forward light scatter, also known as retinal straylight. Forward light scatter originates predominantly in the cornea (approximately 30\%) [8] and the crystalline lens [9]. The forward light scatter within the retina is believed to be negligible by comparison. The backscatter from the retina and, in particular, the deeper structures involving the retinal pigment epithelium can be quite significant in older individuals, but this light is less useful in decreasing the effective retinal image contrast because of the directional sensitivity of cones. Significant deviations from normal cone alignment towards the centre of the pupil have, however, been reported in myopia [10].

Forward light scatter produces a veil on the retina, which in turn contributes most to reduced retinal image contrast. Scattered light gives rise to 'disability glare', haloes [11], in the case of bright single light sources, and can adversely affect 
many visual tasks, particularly those requiring the detection of fine spatial detail. In this sense, changes in retinal image as a result of forward light scatter in the eye are equivalent to low pass spatial filtering with higher spatial frequencies being affected most.

Any structural changes which disturb the normal functioning of the cornea and lens can cause increased scattered light, for example, early stage cataract, corneal oedema and refractive surgery $[10,12,13]$. Whilst the effects of scattered light on retinal image contrast can be significant, Snellen acuity (measured with close to $100 \%$ contrast optotypes) remains relatively unaffected and explains why scattered light is often overlooked during routine clinical examination [14].

Only a handful of studies have investigated the link between forward light scatter and refractive error; the findings of which have been inconsistent. Rozema et al. [15] used the single ring, C-Quant straylight metre (Oculus Optikgeräte, Wetzlar, Germany) to measure straylight parameters in a large cohort $(n=518$ eyes, mean participant age $\pm \mathrm{sd}, 39.7 \pm 13.2$, range 8.5 to 78 years) with reference to axial length (mean \pm sd, $23.9 \pm 1.3 \mathrm{~mm}$, range 19.85-28.70) and mean spherical refractive error (mean $\pm \mathrm{sd},-1.50 \pm 2.90$, range -10.75 to 8.4 D). The study reported a significant adverse effect of both increasing axial length and myopic refractive error on retinal straylight. More recently, Guber et al. [16] measured retinal straylight using the C-Quant on the dominant eye of 45 young adults (mean age $\pm \mathrm{sd}, 33.13 \pm 10.25$ years; range, 21 to 59 years) with an average mean spherical error of $-1.26 \pm$ $1.80 \mathrm{D}$. Refractive errors were categorized as emmetropia ( $n=$ 14); myopia $(n=16)$; hyperopia $(n=8)$ and astigmatism $(n=$ 7). Specifically, myopic error ranged from $-1.25 \mathrm{D}$ to -5.25 D. Whilst Guber et al. did not find a statistically significant effect of refractive error on straylight, the authors do, however, note an observed reduction in straylight in myopes compared with emmetropes, although the effect failed to reach statistical significance. The Optical Quality Analysis System (OQAS), which is based on the double-pass technique, has also been used to estimate intraocular light scatter with respect to refractive error [17, 18]. Whilst Miao et al. [18] noted an increase in objective scatter index with increasing myopia, MartínezRoda et al. [17] did not; a finding which was, in part, attributed to refractive error differences between cohorts.

In this study, we aim to investigate the effects of refractive error and axial length on a range of forward light scatter parameters. The experiments were carried out using the City University, Light Scatter (LS) test (City Occupational Ltd., London, UK) which employs five extended annuli as light scatter sources and uses a flicker-nulling method, similar to that described by Van Den Berg and Spekreijse [19] to measure both the amount and angular distribution of scattered light in the eye [20].

The LS test allows complete assessment of light scatter parameters, including the integral of the light scatter function and the 'volume' of scattered light expected from a point source. Other tests of light scatter employ a single ring of fixed angular subtense and calculate the same parameters by assuming that the angular distribution of scattered light remains unchanged and equal to that expected for the normal eye.

\section{Materials and methods}

The study was approved by the Optometry departmental proportionate ethics review committee of City University of London. All aspects of the investigation were conducted in line with the tenets of the Declaration of Helsinki. Informed consent was obtained from all individual participants included in the study.

Young-adult participants (18-25 years) were recruited from a university staff-student body. Thirteen participants fulfilled the selection criteria and were included in the study. Insufficient information was available on the magnitude of the effects and the expected variability to carry out any justifiable power calculation to assess the minimum sample size. It was therefore decided to examine as many subjects as possible within the restricted time available in the hope that the expected effects would be sufficiently robust, even when only a small number of subjects could be examined within the time available for the project. High astigmatism $(\geq 1.50 \mathrm{DC})$ and individuals with any signs of ocular pathologies were not included in the study. Objective measurements of noncycloplegic refractive error were obtained using the WAM5500 open-view binocular autorefractor (Ryusyo Industrial Co. Ltd., Osaka, Japan). Measurements were expressed as mean spherical error (MSE, in D). Axial length (AL) was measured using the Topcon Aladdin biometer (Topcon, UK).

Each participant carried out assessment of forward light scatter using the City University, LS test; habitual refractive corrections, where appropriate, were worn throughout. The LS test includes a 'learning' task which each participant undertakes prior to the experiment. A dark disc of $0.8^{\circ}$ diameter is always present at the centre of each glare source together with a glare source annulus of varying size. The stimulus consists of a glare source burst of $7.5-\mathrm{Hz}$ rapid flicker that lasts for $\sim 400 \mathrm{~ms}$. The test employs the flicker-nulling technique to measure two zero-flicker thresholds for each annulus. Interleaved staircases are employed, and the participant's task is to report when flicker is no longer detected. The first threshold represents the luminance modulation depth of the dark disc that is just below that caused by scattered light. The second threshold corresponds to a disc modulation amplitude that is higher than that caused by scattered light when the participant also just fails to detect rapid flicker. The difference between the two thresholds is a measure of the participant's sensitivity to flicker for the stimulus conditions employed in the LS test. The midpoint between the two thresholds is taken 
as a best estimate of the luminance of the external source that generates the same retinal illuminance as the glare source. When all the measurements are completed and the veiling luminance estimated for each annulus, the program computes the best parameters, $k$, and, $n$, in the light scatter equation, $\mathrm{L}_{\mathrm{S}} /$ $\mathrm{E}=\mathrm{k} \cdot \theta^{-\mathrm{n}}$, to fit the measured data. $E$ represents the illuminance level generated by the glare source (in lumens $/ \mathrm{m}^{2}$ ) in the plane of the pupil which is known for each annulus; $k$ is the eye specific, light scatter parameter; and $n$ is the scatter index which determines the angular distribution of forward light scatter in the eye [21].

Numerical methods are used in the LS test to compute the area under the light scatter function (i.e. parameter $k^{\prime}$ ) from 2 to $90^{\circ}$ as well as the volume of 'scatted light' (expected from 2 to $90^{\circ}$ ) one obtains when rotating the line scatter function through $360^{\circ}$ (i.e. parameter $k^{\prime \prime}$ ). The program then displays the fitted light scatter function and the computed parameters (for graphical output of the results see LS test: https:// www.city.ac.uk/avot).

\section{Statistical analyses}

Statistical analyses were conducted using IBM SPSS Statistics (IBM UK Ltd. Portsmouth, UK). 0.05 was taken as the level of statistical significance was for all comparisons. Linear regression fits were used to test for significant correlations between each of the light scatter parameters $\left(k^{\prime}, k^{\prime \prime}, n\right)$ and AL and MSE. Given the strong correlation between AL and MSE, partial correlations were used to control for this covariance. Since light scatter functions were tested binocularly, data for both eyes are shown.

\section{Results}

Mean spherical errors were (MSE \pm sd, D) $-1.69 \pm 2.02$ (range 0.38 to -4.75 ) for the right eye and $-1.91 \pm 1.94$ (range 0.50 to -4.63 ) for the left eye with anisometropia $\leq$
1.25 D. Mean axial lengths $(\mathrm{mm} \pm \mathrm{sd})$ were $24.24 \pm 0.95$ and $24.13 \pm 1.01$ for the right and left eyes, respectively.

A Pearson correlation test revealed significant correlation between MSE and AL for both the right and left eyes $(p<0.01$ for both) indicating that any myopia was axial in nature. A paired samples $t$ test showed no significant difference between eyes for axial length or MSE ( $p>0.05$ for both).

Mean values \pm standard deviations for $k, k^{\prime}, k^{\prime \prime}$, and $n$ were $16.82 \pm 6.35$ (CI 12.98-20.66); 6.23 \pm 1.44 (CI 5.36-7.10); $273.77 \pm 98.17$ (CI 214.44-333.10); and 2.16 \pm 0.24 (CI 2.02-2.30), respectively.

\section{MSE}

A significant correlation was found between increasing myopic MSE and increase in both the integral of light scatter $\left(k^{\prime}\right)$ (RE, $p<0.01 ; r^{2} 0.67$; LE, $\left.p<0.01 ; r^{2}=0.66\right)$ and the total volume of light scatter $\left(k^{\prime \prime}\right)\left(\mathrm{RE}, p=0.02 ; r^{2} 0.41\right.$; LE, $p=$ $\left.0.01, r^{2}=0.46\right)$. Since the correlation between AL and MSE is significant, it remains uncertain as to whether the increased amount of light scattered in the eye can be attributed to increasing myopic MSE or simply to increased AL. When controlling for axial length as a covariate in the statistical analysis, the only remaining statistically significant correlation is with $k^{\prime}$ (RE, $p<0.05 ; r=0.64$; LE, $p<0.05, r=0.66$ ) (see Figs. 1 and 2 ). There was no significant relationship found between the angular distribution of light scatter (parameter $n$ ) and refractive error ( $p>0.05$ for both eyes), or refractive error and the straylight parameter $k(p>0.05)$. Use of a quadratic (2nd order polynomial) fits for Fig. 1 ( $k^{\prime}$ vs. MSE) showed the $r^{2}$ value improved to 0.72 for both eyes.

\section{Axial length}

For axial length, significant correlations were noted between a longer axial length and increased in the integral of light scatter $\left(k^{\prime}\right)\left(\mathrm{RE}, p=0.01 ; r^{2}=0.41\right.$; LE, $\left.p=0.02, r^{2}=0.46\right)$ (see Fig. 3); however, the effect disappeared for both the right and
Fig. 1 Changes in $k^{\prime}$ with mean spherical error (MSE)
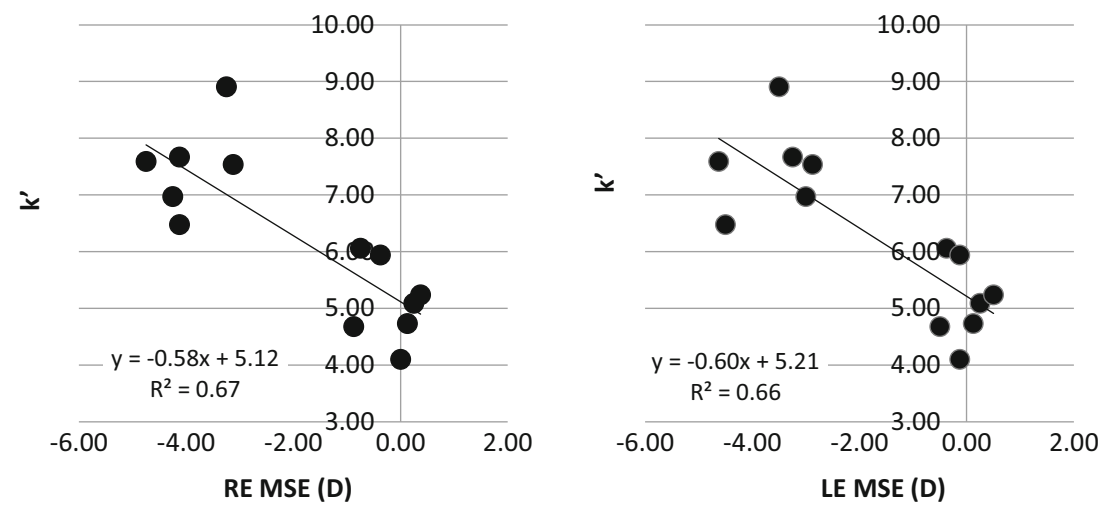
Fig. 2 Changes in $k^{\prime \prime}$ with mean spherical error (MSE)

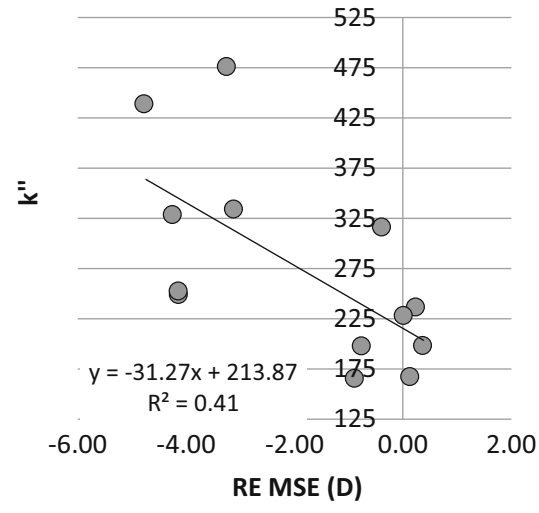

left eyes when controlling for MSE as a covariate ( $p>0.05$ for both eyes). This analysis is equivalent to that described for MSE. No significant relationships were found between $\mathrm{n}$ and axial length ( $p>0.05$ both eyes), or between axial length and the total volume of light scatter, $k^{\prime \prime}$ (see Fig. 4). There was also no significant relationship between axial length and the straylight parameter, $k$ ( $p>0.05$ both eyes).

\section{Discussion}

In general, the results are in agreement with the findings published by Rozema et al. [15]. Increase in myopia and the (inevitable) increase in the axial length of the eye were correlated with an increase in the total amount of light scatter in the eye. This is not surprising since either an increase in refractive error or the axial length of the eye may correlate with structural changes in the cornea or some other structure in the eye that causes the measured increase in the amount of scattered light. When the refractive error was controlled, axial length failed to show a good correlation with the amount of scatter light in myopic eyes. The results reported here are different than those reported by Guber et al. [16], who found emmetropes generally showed more light scatter than myopes.
Our data also show that whilst refractive error affected significantly the amount of scattered light in the eye, in contrast, the angular distribution of scattered light as reflected in the straylight parameter, $n$, showed no significant correlation with either axial length or MSE.

Due to the paucity of work in this field, the source of variation in light scatter with respect to refractive error remains unclear. The reasons why the various studies that examined light scatter in myopes produced inconsistent results [15-18] also remain unexplained, although the use of different light scatter measuring apparatus and techniques may have played a part.

Based on what is known to cause forward light scatter in the eye, the results from this study suggest that myopic eyes may develop greater structural changes in either the cornea, the lens, or both when compared with emmetropic eyes. Participants with cataracts, history of refractive surgery, and corneal disease were excluded from the study. Any structural changes in the cornea and/or lens in the participants investigated are therefore likely to be physiological. Whilst it is known that there may be biomechanical changes to the cornea in myopia [22-24], there is little data about the corneal cellular structure with respect to either refractive error or axial length. That axial length failed to correlate with the measured light
Fig. 3 Changes in $k^{\prime}$ with axial length
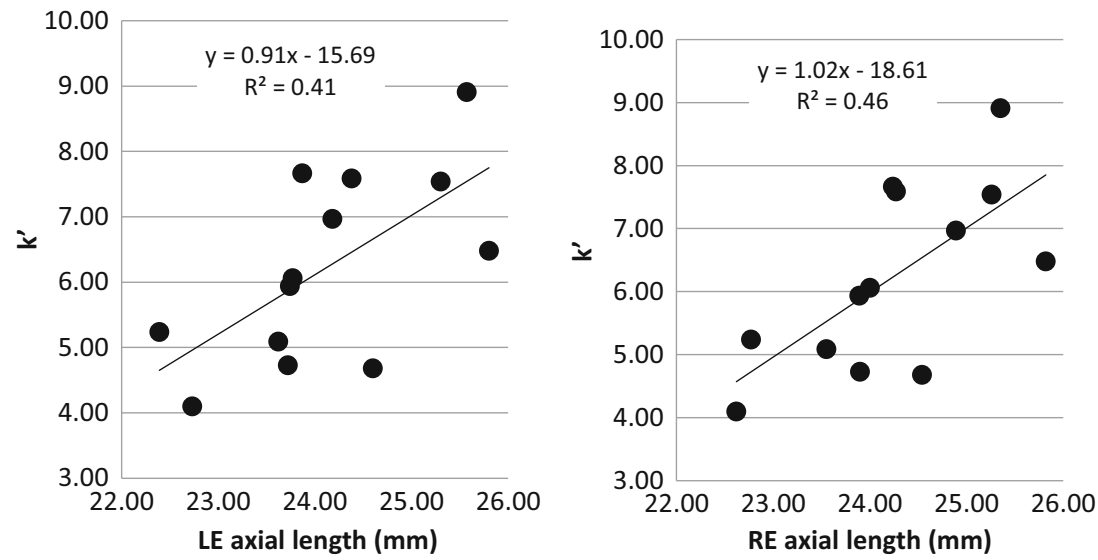
Fig. 4 Changes in $k^{\prime \prime}$ with axial length $(p>0.05)$

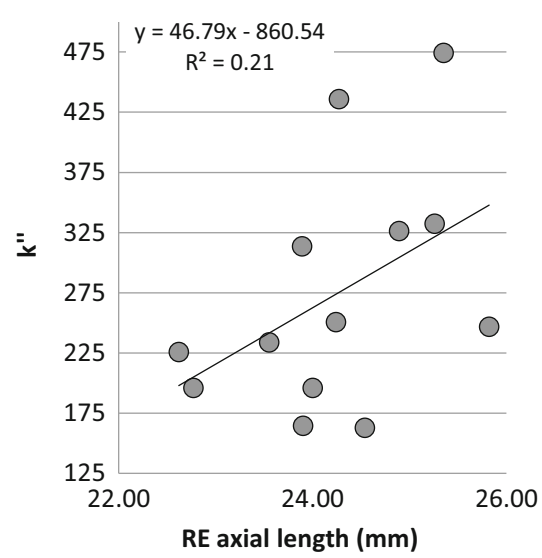

scatter parameters lends support to the view that the anterior eye, and not the retina, contributes most of the measured variation in scattered light.

A limitation of the current study could be that assessment of the light scatter function was undertaken binocularly. If, however, the two eyes differed significantly in their light scattering properties, the measurement of scattered light using the flicker-nulling test would have been very difficult since no single null point would apply to both eyes. The participants, however, had no difficulty in nulling the flicker caused by scattered light, even when the test was carried out binocularly. This observation suggests that the differences in light scatter between the two eyes must have been small. This is unsurprising, given the small anisometropic differences between the two eyes (i.e. $\leq 1.25 \mathrm{D}$ ). One can also argue that if the amount of scattered light measured relates directly to refractive error, the relationship between the mean (inter-eye) refractive error and the light scatter parameters should also be examined. In addition to examining the relationship between refractive error and the amount of scattered light for each eye separately, we also investigated what happens when the refractive errors were averaged. Each of the three analyses showed the same correlations, which suggest that the differences in light scatter parameters between the two eyes for each study participant were small.

One theoretical confounding factor in studies involving myopic participants may be the long-term use of contact lenses and associated corneal oedema. In our cohort, this is unlikely to be the case; modern contact lens materials generally offer high oxygen permeability, thus minimizing the likelihood of corneal oedema. Nevertheless, light scatter is also reported to increase in individuals with corneal surface disturbances caused by 'dry eye' or superficial punctuate keratitis [25] which are likely to be more prevalent amongst contact lens wearers and/or myopes [26]. Further, wearing a contact lens during the light scatter test can, in itself, adversely affect the results $[13,27]$. Another potential source of bias may be the use of spectacles; if lenses were scratched or insufficiently cleaned, these factors may have contributed a small amount to the increased light scatter observed in myopes.

Pupil size may be affected by refractive status [28]. Although the measured light scatter parameters are independent of pupil size [29], this is only so when the light scattering is uniform over the pupil. Several reports have linked retinal straylight to pupil size, and these suggest that forward light scatter may not be uniform over the pupil [30-32].

In summary, the preliminary data from this study provide evidence that the total amount of scattered light in myopic eyes correlates with the magnitude of refractive error. Further studies are needed to characterize how changes in the anterior media of the eye, and a wider range of refractive errors, may affect forward light scatter.

\section{Compliance with ethical standards}

Conflict of interest John Barbur is an inventor of AVOT tests (some employed in this study); an employee of City, University of London; and a director of City Occupational Ltd. (a City University spin out company setup to manufacture and supply AVOT tests). All the other authors declare that they have no conflict of interest.

Ethical approval All procedures performed in studies involving human participants were in accordance with the ethical standards of the institutional and/or national research committee and with the 1964 Helsinki declaration and its later amendments or comparable ethical standards. Informed consent was obtained from all individual participants included in the study.

Open Access This article is licensed under a Creative Commons Attribution 4.0 International License, which permits use, sharing, adaptation, distribution and reproduction in any medium or format, as long as you give appropriate credit to the original author(s) and the source, provide a link to the Creative Commons licence, and indicate if changes were made. The images or other third party material in this article are included in the article's Creative Commons licence, unless indicated otherwise in a credit line to the material. If material is not included in the article's Creative Commons licence and your intended use is not permitted by statutory regulation or exceeds the permitted use, you will need to obtain permission directly from the copyright holder. To view a copy of this licence, visit http://creativecommons.org/licenses/by/4.0/. 


\section{References}

1. Ho WC, Ng YF, Chu PHW, Fong YY, Yip KS, Kee CS, Chan HHL (2011) Impairment of retinal adaptive circuitry in the myopic eye. Vis Res 51(3):367-375

2. Chen JC, Brown B, Schmid KL (2006) Delayed mfERG responses in myopia. Vis Res 46(8-9):1221-1229

3. Rudnicka AR, Edgar DF (1996) Automated static perimetry in myopes with peripapillary crescents - part II. Ophthalmic Physiol Opt 16(5):416-429

4. Kawabata H, Adachi-Usami E (1997) Multifocal electroretinogram in myopia. Invest Ophthalmol Vis Sci 38(13):2844-2851

5. Chui TYP, Song H, Burns SA (2008) Individual variations in human cone photoreceptor packing density: variations with refractive error. Invest Ophthalmol Vis Sci 49(10):4679-4687

6. Choi SS, Enoch JM, Kono M (2004) Evidence for transient forces/ strains at the optic nerve head in myopia: repeated measurements of the Stiles-Crawford Effect of the First Kind (SCE-I) over time. Ophthalmic Physiol Opt 24(3):194-206

7. Westheimer G (1968) Entoptic visualization of Stiles-Crawford Effect: an indicator of eyeball shape. Arch Ophthalmol 79(5): 584-588

8. Vos JJ, Boogaard J (1963) Contribution of the cornea to entoptic scatter. JOSA 53(7):869-873

9. Bettelheim FA, Ali S (1985) Light scattering of normal human lens III. Relationship between forward and back scatter of whole excised lenses. Exp Eye Res 41(1):1-9

10. Choi SS, Garner LF, Enoch JM (2003) The relationship between the Stiles-Crawford effect of the first kind (SCE-I) and myopia. Ophthalmic Physiol Opt 23(5):465-472

11. Spadea L, Maraone G, Verboschi F, Vingolo EM, Tognetto D (2016) Effect of corneal light scatter on vision: a review of the literature. Int J Ophthalmol 9(3):459

12. Fujikado T, Kuroda T, Maeda N, Ninomiya S, Goto H, Tano Y et al (2004) Light scattering and optical aberrations as objective parameters to predict visual deterioration in eyes with cataracts. J Cataract Refract Surg 30(6):1198-1208

13. Elliott DB, Mitchell S, Whitaker D (1991) Factors affecting light scatter in contact lens wearers. Optometry Vis Sci 68(8):629-633

14. Rubin, G. S., Adamsons, I. A., \& Stark, W. J. (1993). Comparison of acuity, contrast sensitivity, and disability glare before and after cataract surgery. Arch Ophthalmol, 111(1), 56-61

15. Rozema JJ, Van den Berg TJ, Tassignon MJ (2010) Retinal straylight as a function of age and ocular biometry in healthy eyes. Invest Ophthalmol Vis Sci 51(5):2795-2799

16. Guber I, Bachmann LM, Guber J, Bochmann F, Lange AP, Thiel MA (2011) Reproducibility of straylight measurement by C-Quant for assessment of retinal straylight using the compensation comparison method. Graefes Arch Clin Exp Ophthalmol 249(9):1367

17. Martínez-Roda JA, Vilaseca M, Ondategui JC, Giner A, Burgos FJ, Cardona G, Pujol J (2011) Optical quality and intraocular scattering in a healthy young population. Clin Exp Optom 94(2):223-229
18. Miao H, Tian M, He L, Zhao J, Mo X, Zhou X (2014) Objective optical quality and intraocular scattering in myopic adults. Invest Ophthalmol Vis Sci 55(9):5582-5587

19. Van Den Berg TJTP, Spekreijse H (1987) Measurement of the straylight function of the eye in cataract and other optical media disturbances by means of a direct compensation method. Invest Ophthalmol Vis Sci 28(suppl):397

20. Hennelly ML, Barbur JL, Edgar DF, Woodward EG (1997) The light scattering characteristics of the eye. Ophthalmic Physiol Opt 17(2):171-171

21. Patterson EJ, Bargary G, Barbur JL (2015) Understanding disability glare: light scatter and retinal illuminance as predictors of sensitivity to contrast. JOSA A 32(4):576-585

22. Lim L, Gazzard G, Chan YH, Fong A, Kotecha A, Sim EL et al (2008) Cornea biomechanical characteristics and their correlates with refractive error in Singaporean children. Invest Ophthalmol Vis Sci 49(9):3852-3857

23. Shen M, Fan F, Xue A, Wang J, Zhou X, Lu F (2008) Biomechanical properties of the cornea in high myopia. Vis Res 48(21):2167-2171

24. Chang SW, Tsai IL, Hu FR, Lin LLK, Shih YF (2001) The cornea in young myopic adults. Br J Ophthalmol 85(8):916-920

25. Koh S, Maeda N, Ikeda C, Asonuma S, Mitamura H, Oie Y, ... \& Nishida K (2014). Ocular forward light scattering and corneal backward light scattering in patients with dry eye. Invest Ophthalmol Vis Sci, 55(10), 6601-6606

26. Ilhan N, Ilhan O, Tuzcu EA, Daglioglu MC, Coskun M, Parlakfikirer N, Keskin U (2014) Is there a relationship between pathologic myopia and dry eye syndrome? Cornea 33(2):169-171

27. Lohmann CP, Fitzke F, O’Brart D, Muir MK, Timberlake G, Marshall J (1993) Corneal light scattering and visual performance in myopic individuals with spectacles, contact lenses, or excimer laser photorefractive keratectomy. Am J Ophthalmol 115(4):444 453

28. Guillon M, Dumbleton K, Theodoratos P, Gobbe M, Wooley CB, Moody K (2016) The effects of age, refractive status, and luminance on pupil size. Optom Vis Sci 93(9):1093

29. Edgar DF, Barbur JL, Woodward EG (1995) Pupil size measurements in relation to light scatter in the eye. Invest Ophthalmol Vis Sci 36(4):S938-S938

30. Franssen L, Tabernero J, Coppens JE, van den Berg TJ (2007) Pupil size and retinal straylight in the normal eye. Invest Ophthalmol Vis Sci 48(5):2375-2382

31. Veraart HG, van den Berg TJ, IJspeert JK, Cardozo OL (1992) Stray light in radial keratotomy and the influence of pupil size and straylight angle. Am J Ophthalmol 114(4):424-428

32. van Gaalen KW, Koopmans SA, Hooymans JM, Jansonius NM, Kooijman AC (2010) Straylight measurements in pseudophakic eyes with natural and dilated pupils: one-year follow-up. J Cataract Refract Surg 36(6):923-928

Publisher's note Springer Nature remains neutral with regard to jurisdictional claims in published maps and institutional affiliations. 